\title{
Besov-type spaces for the $\kappa$-Hankel wavelet transform on the real line
}

https://doi.org/10.1515/conop-2020-0117

Received March 1, 2021; accepted July 9, 2021

Abstract: In this paper, we shall introduce functions spaces as subspaces of $L_{\kappa}^{p}(\mathbb{R})$ that we call Besov- $\kappa$-Hankel spaces and extend the concept of $\kappa$-Hankel wavelet transform in $L_{\kappa}^{p}(\mathbb{R})$ space. Subsequently we will characterize the Besov- $\kappa$-Hankel space by using $\kappa$-Hankel wavelet coefficients.

Keywords: Besov $\kappa$-Hankel space,Continuous $\kappa$-Hankel wavelet transform, $\kappa$-Hankel transform, $\kappa$-Hankel convolution

MSC: 33A40, 44A05, 42C40

Dedicated to the memory of Prof. R. S. Pathak

\section{Introduction}

Besov spaces $B_{\alpha}^{p, q}(\mathbb{R})$ are subspaces of $L^{p}(\mathbb{R})$, having functions of smoothness $\alpha$ and q gives a finer graduation to the smoothness. It is extension of classical Sobolev and Hölder spaces. It is also expressed as interpolation space lies in between two Sobolev spaces $H_{p}^{x}$ and $H_{p}^{y}(1 \leq p, q \leq \infty)$ with $\alpha=(1-\beta) x+\beta y \quad ; \alpha, x, y \in \mathbb{R}, \beta \in(0,1)$.

The Besov spaces $B_{\alpha}^{p, q}(\alpha \in \mathbb{R}$ and $1 \leq p, q \leq \infty)$ were recognized in about sixty ${ }^{\text {th }}$ decade of ninteen ${ }^{\text {th }}$ century [3, 4]. They were generalized in mid of seventies by various authors in different directions with different ideas. The classical definition of Besov spaces depends on the modulus of smoothness [11, 13]. The Littlewood-Paley theory interlink Besov spaces with the fourier transform. Michael Frazier and Björn Jawerth [6] characterise Besov spaces with the help of Calderon's formula while Dang Vu Giang and Ferenc Moricz [7] characterise Besov spaces in terms of it's Riesz mean and Dirichlet integral. In 1996, Valerie Perrier and Claude Basdevant [10] characterise Besov spaces by the behavior of the continuous wavelet coefficients for $\alpha \in \mathbb{R}^{+}, \quad \alpha \notin \mathbb{Z}^{+}$.

Jorge J. Betancor and L. Rodríguez-Mesa [5] pave the way for exploration of Besov-Hankel spaces and characterized by mean of the Bochner-Riesz mean and the partial Hankel integrals. Recently Salem Ben Saïd, Mohamed Amine Boubatra, Mohamed Sifi [2] come out with deformed Besov-Hankel spaces and characterised it in terms of the deformed Bochner-Riesz means and the deformed partial Hankel integral.

Hatem Mejjaoli, khalifa Trimèche [8] presented $\kappa$-Hankel wavelet transform in the year 2020. Using the approach of Betancor et al. [5] and Ben Saïd et al. [2], we define Besov $\kappa$-Hankel space and by exploiting the technique of Perrier et al. [10] we characterise Besov $\kappa$-Hankel space with the help of continuous $\kappa$-Hankel wavelet transform.

Present paper is organized in following manner: section 1 is introductory, in which we define the development from Besov space to Besov $\kappa$-Hankel space with the help of $\kappa$-Hankel wavelet and it's characterisation

*Corresponding Author: Ashish Pathak: Department of Mathematics, Institute of Science, Banaras Hindu University, Varanasi-221005, India, E-mail: ashishpathak@bhu.ac.in

Shrish Pandey: Department of Mathematics, Institute of Science, Banaras Hindu University, Varanasi-221005, India, E-mail: shrish20594@gmail.com 
with time period. section 2 is preliminary, in which we recall some properties of $\kappa$-Hankel transform , Besov $\kappa$-Hankel space and continuous $\kappa$-Hankel wavelet transform. Section 3 is related the continuous $\kappa$-Hankel wavelet transform in $L_{\kappa}^{p}(\mathbb{R})$. In the section 4, we characterize Besov $\kappa$-Hankel norms in terms of continuous $\kappa$-Hankel wavelet transform.

\section{Preliminary}

In this paper, we denotes the weighted $L_{\kappa}^{p}(\mathbb{R})$ space as

$$
\|f\|_{L_{\kappa}^{p}}=\|f\|_{p}=\left(\int_{\mathbb{R}}|f(x)|^{p} d \sigma_{\kappa}(x)\right)^{\frac{1}{p}},(1 \leq p<\infty),
$$

where $d \sigma_{\kappa}(x)=|x|^{2 \kappa-1} d x, \kappa \geq \frac{1}{2}$.

$$
\|f\|_{L_{\kappa}^{\infty}}=\|f\|_{\infty}=\operatorname{esssup}|f(x)| .
$$

The $\kappa$-Hankel transformation of the function $f \in L_{\kappa}^{1}(\mathbb{R})$ for order $\kappa \geq \frac{1}{2}$ is defined as see [8]

$$
\left(\Im_{\kappa} f\right)(\mu)=\hat{f}(\mu):=\frac{1}{\rho_{\kappa}} \int_{\mathbb{R}} \mathcal{B}_{\kappa}(\mu, x) f(x) d \sigma_{\kappa}(x), \quad x \in \mathbb{R},
$$

where

$$
\rho_{\kappa}=\int_{\mathbb{R}} e^{-|x|} d \sigma_{\kappa}(x)=2 \Gamma(2 \kappa)
$$

and $\mathcal{B}_{\kappa}(\mu, x)$ is the $\kappa$-Hankel kernel given as

$$
\mathcal{B}_{\kappa}(\mu, x)=j_{2 \kappa-1}(2 \sqrt{|\mu x|})-\frac{\mu x}{2 \kappa(2 \kappa+1)} j_{2 \kappa+1}(2 \sqrt{|\mu x|}) .
$$

Here

$$
j_{\lambda}(\omega)=\Gamma(\lambda+1)\left(\frac{\omega}{2}\right)^{-\lambda} J_{\lambda}(\omega)=\Gamma(\lambda+1) \sum_{n=0}^{\infty} \frac{(-1)^{n}}{n ! \Gamma(\lambda+n+1)}\left(\frac{\omega}{2}\right)^{2 n}
$$

denote the normalized Bessel function of index $\lambda$.

If $\hat{f} \in L_{\kappa}^{1}(\mathbb{R})$, then the inverse of $\kappa$-Hankel transformations is given by

$$
f(x):=\frac{1}{\rho_{\kappa}} \int_{\mathbb{R}} \mathcal{B}_{\kappa}(\mu, x) \hat{f}(\mu) d \sigma_{\kappa}(\mu), \quad x \in \mathbb{R} .
$$

Also, Parseval's formula of the $\kappa$-Hankel transformation for $f, g \in L_{\kappa}^{1}(\mathbb{R}) \cap L_{\kappa}^{2}(\mathbb{R})$ is given by

$$
\int_{\mathbb{R}} \hat{f}(\mu) \overline{\hat{g}(\mu)} d \sigma_{\kappa}(\mu)=\int_{\mathbb{R}} f(x) \overline{g(x)} d \sigma_{\kappa}(x) .
$$

By denseness and continuity the Parseval's formula can be extended to all $f, g \in L_{\kappa}^{2}$. Hence $\Im_{\kappa}$ is isometry on $L_{\kappa}^{2}(\mathbb{R})$.

If $f, g \in L_{\kappa}^{2}(\mathbb{R})$, then the convolution associated with the $\kappa$-Hankel transform is defined as see [1]

$$
\left(f \#_{\kappa} g\right)(x)=\frac{1}{\rho_{\kappa}} \int_{\mathbb{R}} f(y) \tau_{x}^{\kappa} g(y) d \sigma_{\kappa}(y),
$$


where the operatore $\tau_{x}^{\kappa}$ is $\kappa$-Hankel translation is given by

$$
f^{\kappa}(x, y)=\tau_{x}^{\kappa} f(y)=\int_{\mathbb{R}} f(z) \mathcal{K}_{\kappa}(x, y, z) d \sigma_{\kappa}(z)
$$

and

$$
\int_{\mathbb{R}} \mathcal{B}_{\kappa}(\mu, z) \mathcal{K}_{\kappa}(x, y, z) d \sigma_{\kappa}(z)=\mathcal{B}_{\kappa}(\mu, y) \mathcal{B}_{\kappa}(\mu, x) .
$$

From (1) and (3), we have

$$
\mathcal{K}_{\kappa}(x, y, z)=\frac{1}{\rho_{\kappa}^{2}} \int_{\mathbb{R}} \mathcal{B}_{\kappa}(\mu, x) \mathcal{B}_{\kappa}(\mu, y) \mathcal{B}_{\kappa}(\mu, z) d \sigma_{\kappa}(\mu)
$$

and moreover,

$$
\begin{gathered}
\int_{\mathbb{R}} \mathcal{K}_{\kappa}(x, y, z) d \sigma_{\kappa}(z)=1 \\
\int_{\mathbb{R}}\left|\mathcal{K}_{\kappa}(x, y, z)\right| d \sigma_{\kappa}(z) \leq M_{\kappa}
\end{gathered}
$$

where $M_{\kappa}$ is independent of $\mathrm{x}$ and y such that $M_{\kappa} \leq 2$ as $\kappa \rightarrow \infty$ whenever $x y<0$ and $M_{\kappa} \leq 3$ as $k \rightarrow \infty$ elsewhere.

$$
\left(f \hat{\#}_{K} g\right)(x)=\hat{f}(x) \hat{g}(x) .
$$

Now, we recall some properties of $\kappa$-Hankel convolution [1] which are useful throught the paper.

Lemma 2.1. Let $f \in L_{\kappa}^{p}(\mathbb{R}), 1 \leq p \leq \infty$. Then we have

$$
\left\|\tau_{x}^{\kappa} f(y)\right\|_{p} \leq M_{\kappa}|| f \|_{p}
$$

Lemma 2.2. Let $f \in L_{\kappa}^{p}(\mathbb{R})$ and $g \in L_{\kappa}^{q}(\mathbb{R}), \frac{1}{p}+\frac{1}{q}=1+\frac{1}{r}$. Then we have

$$
\left\|f \#_{\kappa} g\right\|_{r} \leq M_{\kappa}|| f|| p\|g\|_{q} .
$$

Definition 2.3. (Besov $\kappa$-Hankel Space): Let measurable function $\phi$ defined on $\mathbb{R}$ belongs to $\mathcal{B H}_{\alpha, \kappa}^{p, q}$ if $\phi \in$ $L_{\kappa}^{p}(\mathbb{R})$ and

$$
\begin{gathered}
\int_{0}^{\infty}\left(h^{-\alpha} w_{p, \kappa}(\phi)(h)\right)^{q} \frac{d h}{h}<\infty \text { for }, 1 \leq p, q<\infty, \\
\operatorname{esssup}_{h>0}\left(h^{-\alpha} w_{p, \kappa}(\phi)(h)\right)<\infty \text { for } q=\infty,
\end{gathered}
$$

where $w_{p, \kappa}(\phi)(h)=:\left\|\tau_{h}^{\kappa} \phi-\phi\right\|_{L_{\kappa}^{p}}, h \in \mathbb{R}^{+}$and $0<\alpha<1$.

\section{$2.1 \kappa$-Hankel wavelet}

Using the properites of $\kappa$-Hankel transform see [8] define the $\kappa$-Hankel wavelet for $\varphi \in L_{\kappa}^{p}(\mathbb{R}), 1 \leq p<\infty$, $b \in \mathbb{R}$ and $a>0$ as

$$
\begin{aligned}
\varphi_{b, a}^{\kappa}(x): & =D_{a} \tau_{b}^{\kappa} \varphi(x) \\
& =D_{a} \varphi^{\kappa}(x, b) \\
& =a^{-2 \kappa} \varphi^{\kappa}\left(\frac{x}{a}, \frac{b}{a}\right) \\
& =a^{-2 \kappa} \int_{\mathbb{R}} \varphi(z) \mathcal{K}_{\kappa}\left(\frac{b}{a}, \frac{x}{a}, z\right) d \sigma_{\kappa}(z)
\end{aligned}
$$


where $D_{a}$ denote the dilation operatore such that

$$
D_{a} \varphi(x, y)=a^{-2 \kappa} \varphi\left(\frac{x}{a}, \frac{y}{a}\right) .
$$

The continuous $\kappa$-Hankel wavelet transform of $f \in L_{\kappa}^{2}(\mathbb{R})$ with respect to a wavelet $\varphi \in L_{\kappa}^{2}(\mathbb{R})$ is defined as [8]

$$
\begin{aligned}
\left(\mathcal{H}_{\varphi}^{\kappa} f\right)(b, a) & :=\frac{1}{\rho_{\kappa}} \int_{\mathbb{R}} f(x) \bar{\varphi}_{b, a}^{\kappa}(x) d \sigma_{\kappa}(x) \\
& =\frac{a^{-2 \kappa}}{\rho_{\kappa}} \int_{\mathbb{R}} \int_{\mathbb{R}} f(x) \bar{\varphi}(z) \mathcal{K}_{\kappa}\left(\frac{b}{a}, \frac{x}{a}, z\right) d \sigma_{\kappa}(z) d \sigma_{\kappa}(x) .
\end{aligned}
$$

Moreover , using (2), we have

$$
\left(\mathcal{H}_{\varphi}^{\kappa} f\right)(b, a)=\left(f \#_{\kappa} \varphi_{a}^{\kappa}\right)(b)
$$

where $\varphi_{a}^{\kappa}(t)=a^{-2 \kappa} \bar{\varphi}(t / a)$

for more about $\kappa$-Hankel wavelet see [8].

\section{The Continuous $\kappa$-Hankel Wavelet Transform in $L_{\kappa}^{p}(\mathbb{R})$}

In this section we extend the concept of $\kappa$-Hankel wavelet transform on $L_{\kappa}^{p}(\mathbb{R})$.

Theorem 3.1. Suppose that a function $\psi \in L_{\kappa}^{2}(\mathbb{R})$ satisfies the admissibility condition

$$
C_{\kappa, \phi}=\int_{0}^{\infty} \omega^{-1}|\hat{\psi}(\omega)|^{2} d \omega<\infty,
$$

where $\hat{\psi}$ denote the $\kappa$-Hankel transform of $\psi$ then continuous $\kappa$-Hankel wavelet transform is a bounded linear operator

$$
L_{\kappa}^{p}(\mathbb{R}) \rightarrow L_{\kappa}^{2}\left(\mathbb{R}^{+}, \frac{d \sigma_{\kappa}(a)}{a^{2 \kappa}}\right) \times L_{\kappa}^{p}(\mathbb{R}),
$$

moreover, for any $f \in L_{\kappa}^{p}(\mathbb{R}), 1<p<\infty$

$$
\|f\|_{L_{\kappa}^{p}(\mathbb{R})} \simeq\left(\int_{-\infty}^{\infty}\left(\int_{0}^{\infty}\left|\left(\mathcal{H}_{\psi}^{\kappa} f\right)(b, a)\right|^{2} \frac{d \sigma_{\kappa}(a)}{a^{2 \kappa}}\right)^{\frac{p}{2}} d \sigma_{\kappa}(b)\right)^{\frac{1}{p}} .
$$

Proof. Let $S_{p}$ denote the space $L_{\kappa}^{2}\left(\mathbb{R}^{+}, \frac{d \sigma_{\kappa}(a)}{a^{2 k}}\right) \times L_{\kappa}^{p}(\mathbb{R})$ associated to the norm

$$
\|f\|_{S_{p}}=\left\{\int_{-\infty}^{\infty}\left(\int_{0}^{\infty}|f(b, a)|^{2} \frac{d \sigma_{\kappa}(a)}{a^{2 \kappa}}\right)^{\frac{p}{2}} d \sigma_{\kappa}(b)\right\}^{\frac{1}{p}} .
$$

If we take $p=2$, then from Plancherel's theorem [8]:

$$
\begin{aligned}
\left\|\left(\mathcal{H}_{\psi}^{\kappa} f\right)\right\|_{S_{2}} & =\left\{\int_{-\infty}^{\infty}\left(\int_{0}^{\infty}\left|\left(\mathcal{H}_{\psi}^{\kappa} f\right)(b, a)\right|^{2} \frac{d \sigma_{\kappa}(a)}{a^{2 \kappa}}\right) d \sigma_{\kappa}(b)\right\}^{\frac{1}{2}} \\
& =\sqrt{C_{\kappa, \psi}}\|f\|_{L_{\kappa}^{2}(\mathbb{R})}
\end{aligned}
$$


where $C_{\kappa, \psi}=\int_{0}^{\infty} \omega^{-1}|\hat{\psi}(\omega)|^{2} d \omega<\infty$, if $\psi$ is real. From singular integral theorem, the operators on $L_{\kappa}^{2}\left(\mathbb{R}^{+}, \frac{d \sigma_{K}(a)}{a^{2 k}}\right)$ holds inequality:

$$
\left\|\mathcal{H}_{\psi}^{K} f\right\|_{S_{p}} \leq C_{p}\|f\|_{L_{\kappa}^{p}(\mathbb{R})} \text { for } 1<p \leq 2,
$$

where the constant $C_{p}$ depends only on $p$ and $\psi($ see [12]). Due to duality the inequality is also valid for $1<$ $p<\infty$. It follows that

$$
\left\{\int_{-\infty}^{\infty}\left(\int_{0}^{\infty}\left|\left(\mathcal{H}_{\psi}^{\kappa} f\right)(b, a)\right|^{2} \frac{d \sigma_{\kappa}(a)}{a^{2 \kappa}}\right)^{\frac{p}{2}} d \sigma_{\kappa}(b)\right\}^{\frac{1}{p}} \leq C_{p}\|f\|_{L_{\kappa}^{p}(\mathbb{R})} .
$$

Conversely suppose that $f \in L_{\kappa}^{2}(\mathbb{R}) \cap L_{\kappa}^{p}(\mathbb{R})$. Since continuous $\kappa$-Hankel wavelet transform is isomerty for every $g \in L_{\kappa}^{2}(\mathbb{R}) \cap L_{\kappa}^{q}(\mathbb{R})$, we can write

$$
\begin{array}{r}
\int_{-\infty}^{\infty} \int_{0}^{\infty}\left(\mathcal{H}_{\psi}^{\kappa} f\right)(b, a) \overline{\left(\mathcal{H}_{\psi}^{\kappa} g\right)(b, a)} a^{-2 \kappa} d \sigma_{\kappa}(a) d \sigma_{\kappa}(b)=C_{\kappa, \psi}\langle f, g\rangle \\
\frac{1}{C_{\kappa, \psi}} \int_{-\infty}^{\infty} \int_{0}^{\infty}\left(\mathcal{H}_{\psi}^{\kappa} f\right)(b, a) \overline{\left(\mathcal{H}_{\psi}^{\kappa} g\right)(b, a)} a^{-2 \kappa} d \sigma_{\kappa}(a) d \sigma_{\kappa}(b)=\int_{-\infty}^{\infty} f(x) \overline{g(x)} d \sigma_{\kappa}(x) .
\end{array}
$$

Now,

$$
\begin{aligned}
\left|\int_{-\infty}^{\infty} f(x) \overline{g(x)} d \sigma_{\kappa}(x)\right| & =\frac{1}{C_{\kappa, \psi}}\left|\int_{-\infty}^{\infty} \int_{0}^{\infty}\left(\mathcal{H}_{\psi}^{\kappa} f\right)(b, a) \overline{\left(\mathcal{H}_{\psi}^{\kappa} g\right)(b, a)} a^{-2 \kappa} d \sigma_{\kappa}(a) d \sigma_{\kappa}(b)\right| \\
& \leq \frac{1}{C_{\kappa, \psi}} \int_{-\infty}^{\infty} \int_{0}^{\infty}\left|\left(\mathcal{H}_{\psi}^{\kappa} f\right)(b, a) \overline{\left(\mathcal{H}_{\psi}^{\kappa} g\right)(b, a)}\right| a^{-2 \kappa} d \sigma_{\kappa}(a) d \sigma_{\kappa}(b)
\end{aligned}
$$

using Schwarz inequality and then Holder's inequality, we have

$$
\begin{array}{r}
\leq \frac{1}{C_{\kappa, \psi}}\left(\int_{-\infty}^{\infty}\left(\int_{0}^{\infty}\left|\left(\mathcal{H}_{\psi}^{\kappa} f\right)(b, a)\right|^{2} a^{-2 \kappa} d \sigma_{\kappa}(a)\right)^{\frac{p}{2}} d \sigma_{\kappa}(b)\right)^{\frac{1}{p}} \\
\quad \times\left(\int_{-\infty}^{\infty}\left(\int_{0}^{\infty}\left|\left(\mathcal{H}_{\psi}^{\kappa} g\right)(b, a)\right|^{2} a^{-2 \kappa} d \sigma_{\kappa}(a)\right)^{\frac{q}{2}} d \sigma_{\kappa}(b)\right)^{\frac{1}{q}},
\end{array}
$$

where $\frac{1}{p}+\frac{1}{q}=1$.

From equation (6), we get

$$
\leq \frac{C_{q}}{C_{\kappa, \psi}}\left(\int_{-\infty}^{\infty}\left(\int_{0}^{\infty}\left|\left(\mathcal{H}_{\psi}^{\kappa} f\right)(b, a)\right|^{2} a^{-2 \kappa} d \sigma_{\kappa}(a)\right)^{\frac{p}{2}} d \sigma_{\kappa}(b)\right)^{\frac{1}{p}}\|g\|_{L_{\kappa}^{q}(\mathbb{R})} .
$$

By Density theorem

$$
\|f\|_{L_{\kappa}^{p}(\mathbb{R})} \leq A\left(\int_{-\infty}^{\infty}\left(\int_{0}^{\infty}\left|\left(\mathcal{H}_{\psi}^{\kappa} f\right)(b, a)\right|^{2} a^{-2 \kappa} d \sigma_{\kappa}(a)\right)^{\frac{p}{2}} d \sigma_{\kappa}(b)\right)^{\frac{1}{p}},
$$

where $A=\frac{C_{q}}{C_{x, \psi}}$. 
Theorem 3.2. (Parseval's formula) Let us assume $\phi_{1} \in L_{\kappa}^{p}(\mathbb{R}), \phi_{2} \in L_{\kappa}^{q}(\mathbb{R})$ with $1<p, q<\infty$ and $\frac{1}{p}+\frac{1}{q}=1$. If $\psi$ is a real wavelet then

$$
\frac{1}{C_{\kappa, \psi}} \int_{-\infty}^{\infty} \int_{0}^{\infty}\left(\mathcal{H}_{\psi}^{\kappa} \phi_{1}\right)(b, a) \overline{\left(\mathcal{H}_{\psi}^{\kappa} \phi_{2}\right)(b, a)} a^{-2 \kappa} d \sigma_{\kappa}(a) d \sigma_{\kappa}(b)=\int_{-\infty}^{\infty} \phi_{1}(x) \overline{\phi_{2}(x)} d \sigma_{\kappa}(x),
$$

where $C_{\kappa, \psi}=\int_{0}^{\infty} \omega^{-1}|\hat{\psi}(\omega)|^{2} d \omega<\infty$ and $\hat{\psi}$ denote the $\kappa$-Hankel transform.

Proof. Let us define bilinear transform $T: L_{\kappa}^{p}(\mathbb{R}) \times L_{\kappa}^{q}(\mathbb{R}) \rightarrow \mathbb{R}$ by

$$
T\left(\phi_{1}, \phi_{2}\right)=\left\langle\left(\mathcal{H}_{\psi}^{\kappa} \phi_{1}\right)(b, a),\left(\mathcal{H}_{\psi}^{K} \phi_{2}\right)(b, a)\right\rangle_{\left(\frac{d \sigma_{K}(a)}{a^{2 k}} d \sigma_{\kappa}(b)\right)} .
$$

Now, applying Hölder's inequality two times we obtain

$$
\begin{aligned}
\left|T\left(\phi_{1}, \phi_{2}\right)\right|= & \left|\left\langle\left(\mathcal{H}_{\psi}^{\kappa} \phi_{1}\right)(b, a),\left(\mathcal{H}_{\psi}^{\kappa} \phi_{2}\right)(b, a)\right\rangle_{\frac{d \sigma_{k}(a)}{a^{2} \kappa} d \sigma_{\kappa}(b)}\right| \\
\leq & \int_{-\infty}^{\infty}\left(\int_{0}^{\infty}\left|\left(\mathcal{H}_{\psi}^{\kappa} \phi_{1}\right)(b, a)\right|^{2} \frac{d \sigma_{\kappa}(a)}{a^{2 \kappa}}\right)^{\frac{1}{2}}\left(\int_{0}^{\infty}\left|\left(\mathcal{H}_{\psi}^{\kappa} \phi_{2}\right)(b, a)\right|^{2} \frac{d \sigma_{\kappa}(a)}{a^{2 \kappa}}\right)^{\frac{1}{2}} d \sigma_{\kappa}(b) \\
\leq & \left(\int_{-\infty}^{\infty}\left(\int_{0}^{\infty}\left|\left(\mathcal{H}_{\psi}^{\kappa} \phi_{1}\right)(b, a)\right|^{2} \frac{d \sigma_{\kappa}(a)}{a^{2 \kappa}}\right)^{\frac{1}{2}} d \sigma_{\kappa}(b)\right)^{\frac{1}{p}} \\
& \times\left(\int_{-\infty}^{\infty}\left(\int_{0}^{\infty}\left|\left(\mathcal{H}_{\psi}^{\kappa} \phi_{2}\right)(b, a)\right|^{2} \frac{d \sigma_{\kappa}(a)}{a^{2 \kappa}}\right)^{\frac{q}{2}} d \sigma_{\kappa}(b)\right)^{\frac{1}{q}}
\end{aligned}
$$

using Theorem 3.1. we have

$$
\left|T\left(\phi_{1}, \phi_{2}\right)\right| \leq A\left\|\phi_{1}\right\|_{L_{\kappa}^{p}(\mathbb{R})}\left\|\phi_{2}\right\|_{L_{\kappa}^{q}(\mathbb{R})} .
$$

Moreover for all $\phi_{1} \in L_{\kappa}^{2}(\mathbb{R}) \cap L_{\kappa}^{p}(\mathbb{R})$ and $\phi_{2} \in L_{\kappa}^{2}(\mathbb{R}) \cap L_{\kappa}^{q}(\mathbb{R})$ we get

$$
T\left(\phi_{1}, \phi_{2}\right)=\left\langle\left(\mathcal{H}_{\psi}^{\kappa} \phi_{1}\right)(b, a),\left(\mathcal{H}_{\psi}^{\kappa} \phi_{2}\right)(b, a)\right\rangle_{\frac{d \sigma_{\kappa}(a)}{a^{2 K}}, d \sigma_{\kappa}(b)}=C_{\kappa, \psi}\left\langle\phi_{1}, \phi_{2}\right\rangle .
$$

From equations (7), (8) and density of spaces $L_{\kappa}^{2}(\mathbb{R}) \cap L_{\kappa}^{p}(\mathbb{R})$ in $L_{\kappa}^{p}(\mathbb{R})$ gives the result.

\subsection{An inversion formula}

Theorem 3.3. Let us consider $\phi \in L_{\kappa}^{p}(\mathbb{R})$ with $1<p<\infty$ and $\psi$ is a real wavelet. Then

$$
\phi(x)=\frac{1}{C_{\kappa, \psi} \rho_{\kappa}} \int_{-\infty}^{\infty} \int_{0}^{\infty}\left(\mathcal{H}_{\psi}^{\kappa} \phi\right)(b, a) \psi_{b, a}^{\kappa}(x) \frac{d \sigma_{\kappa}(a)}{a^{2 \kappa}} d \sigma_{\kappa}(b) .
$$

The equality holds in $L_{\kappa}^{p}(\mathbb{R})$ sense and the integral of right hand side have to be taken in the sense of distributions.

Proof. The proof followed from theorem 3.2.

\section{Characterization of Besov $\boldsymbol{\kappa}$-Hankel Norms}

In present section, By using the above results, we characterize the Besov $\kappa$-Hankel norms associated with the $\kappa$-Hankel wavelet transform. 
Theorem 4.1. Let $f \in \mathcal{B H}_{\alpha, \kappa}^{p, q}(\mathbb{R})(p, q \geq 1,0<\alpha<1)$ and $\psi, z^{\alpha} \psi \in L_{\kappa}^{1}(\mathbb{R})$, then the wavelet transform of function $f$ holds following conditions:

$$
\begin{gathered}
\text { if } q<\infty, \quad \int_{0}^{\infty}\left[a^{-\alpha}\left\|\left(\mathcal{H}_{\psi}^{\kappa} f\right)(., a)\right\|_{L_{\kappa}^{p}}\right]^{q} \frac{d a}{a}<\infty \\
\text { if } q=\infty, \quad a \rightarrow a^{-\alpha}\left\|\left(\mathcal{H}_{\psi}^{\kappa} f\right)(., a)\right\|_{L_{\kappa}^{p}} \in L_{\kappa}^{\infty}\left(\mathbb{R}^{+}\right) .
\end{gathered}
$$

Moreover the function $a \rightarrow a^{-\alpha}\left\|\left(\mathcal{H}_{\psi}^{\kappa} f\right)(., a)\right\|_{L_{\kappa}^{p}} \in L_{\kappa}^{q}\left(\mathbb{R}^{+}, \frac{d a}{a}\right)$.

Proof. By the definition of continuous $\kappa$-Hankel wavelet transform and equation (4), we have

$$
\begin{aligned}
\left(\mathcal{H}_{\psi}^{\kappa} f\right)(b, a) & =\frac{1}{\rho_{\kappa}} \int_{-\infty}^{\infty} f(x) \overline{\psi_{b, a}^{\kappa}(x)} d \sigma_{\kappa}(x) \\
& =\frac{1}{\rho_{\kappa}} \int_{-\infty}^{\infty} f(x)\left(\int_{-\infty}^{\infty} a^{-2 \kappa} \mathcal{K}_{\kappa}\left(\frac{b}{a}, \frac{x}{a}, z\right) \overline{\psi(z)} d \sigma_{\kappa}(z)\right) d \sigma_{\kappa}(x) \\
& =\frac{1}{\rho_{\kappa}} \int_{-\infty}^{\infty} \overline{\psi(z)}\left(\int_{-\infty}^{\infty} a^{-2 \kappa} \mathcal{K}_{\kappa}\left(\frac{b}{a}, \frac{x}{a}, z\right) f(x) d \sigma_{\kappa}(x)\right) d \sigma_{\kappa}(z) \\
& =\frac{1}{\rho_{\kappa}} \int_{-\infty}^{\infty} \overline{\psi(z)}\left(\int_{-\infty}^{\infty} \mathcal{K}_{\kappa}(b, x, a z) f(x) d \sigma_{\kappa}(x)\right) d \sigma_{\kappa}(z) \\
& =\frac{1}{\rho_{\kappa}}\left\{\int_{-\infty}^{\infty}\left(\tau_{a z}^{\kappa} f\right)(b) \overline{\psi(z)} d \sigma_{\kappa}(z)-\int_{-\infty}^{\infty} f(b) \overline{\psi(z)} d \sigma_{\kappa}(z)\right\} \\
& =\frac{1}{\rho_{\kappa}} \int_{-\infty}^{\infty} \overline{\psi(z)}\left(\left(\tau_{a z}^{\kappa} f\right)(b)-f(b)\right) d \sigma_{\kappa}(z) .
\end{aligned}
$$

Taking $L_{\kappa}^{p}-$ norm of the wavelet coefficient

$$
\left\|\left(\mathcal{H}_{\psi}^{\kappa} f\right)(b, a)\right\|_{L_{\kappa}^{p}}=\frac{1}{\rho_{\kappa}}\left\{\int_{-\infty}^{\infty}\left\{\left|\int_{-\infty}^{\infty} \overline{\psi(z)}\left(\left(\tau_{a z}^{\kappa} f\right)(b)-f(b)\right) d \sigma_{\kappa}(z)\right|^{p}\right\} d \sigma_{\kappa}(b)\right\}^{\frac{1}{p}} .
$$

Using Minkowski inequality of integrability for $p \neq \infty$

$$
\left\|\left(\mathcal{H}_{\psi}^{\kappa} f\right)(b, a)\right\|_{L_{\kappa}^{p}} \leq \frac{1}{\rho_{\kappa}} \int_{-\infty}^{\infty}\left\{\int_{-\infty}^{\infty}\left|\left(\tau_{a z}^{\kappa} f(b)-\left.f(b)\right|^{p} d \sigma_{\kappa}(b)\right\}^{\frac{1}{p}}\right| \psi(z) \mid d \sigma_{\kappa}(z) .\right.
$$

Suppose that $q<\infty$ and integrating w.r.t. $a$, we get

$$
\int_{0}^{\infty}\left[a^{-\alpha}\left\|\mathcal{H}_{\psi}^{\kappa} f(b, a)\right\|_{L_{\kappa}^{p}}\right]^{q} \frac{d a}{a} \leq \frac{1}{\rho_{\kappa}} \int_{0}^{\infty}\left[a^{-\alpha} \int_{-\infty}^{\infty}|\psi(z)| \omega_{p, \kappa}(f, a z) d \sigma_{\kappa}(z)\right]^{q} \frac{d a}{a} .
$$

Again using Minkowski integrabilty inequality

$$
\int_{0}^{\infty}\left[a^{-\alpha}\left\|\mathcal{H}_{\psi}^{\kappa} f(b, a)\right\|_{L_{\kappa}^{p}}\right]^{q} \frac{d a}{a} \leq \frac{1}{\rho_{\kappa}}\left[\int_{-\infty}^{\infty}|\psi(z)| d \sigma_{\kappa}(z)\left\{\int_{0}^{\infty}\left(a^{-\alpha} \omega_{p, \kappa}(f, a z)\right)^{q} \frac{d a}{a}\right\}^{\frac{1}{q}}\right]^{q} .
$$


Applying change of variable $h=a z$

$$
\begin{aligned}
& =\frac{1}{\rho_{\kappa}}\left[\int_{-\infty}^{\infty} z^{\alpha}|\psi(z)| d \sigma_{\kappa}(z)\left\{\int_{0}^{\infty}\left(h^{-\alpha} \omega_{p, \kappa}(f, h)\right)^{q} \frac{d h}{h}\right\}^{\frac{1}{q}}\right]^{q} \\
& \leq \frac{1}{\rho_{\kappa}}\left\{\int_{-\infty}^{\infty} z^{\alpha}|\psi(z)| d \sigma_{\kappa}(z)\right\}^{q} \times\left\{\int_{0}^{\infty}\left(h^{-\alpha} \omega_{p, \kappa}(f, h)\right)^{q} \frac{d h}{h}\right\} \\
& =\frac{1}{\rho_{\kappa}}\left\{\int_{-\infty}^{\infty}\left|z^{\alpha} \psi(z)\right| d \sigma_{\kappa}(z)\right\}^{q} \times\left\{\int_{0}^{\infty}\left(h^{-\alpha} \omega_{p, \kappa}(f, h)\right)^{q} \frac{d h}{h}\right\} \\
& <\infty .
\end{aligned}
$$

If $q=\infty$ the hypothesis on $f$ says that $h^{-\alpha} \omega_{p, \kappa}(f, h) \in L_{\kappa}^{\infty}\left(\mathbb{R}^{+}\right)$, so

$$
\begin{aligned}
\left\|\left(\mathcal{H}_{\psi}^{\kappa} f\right)(b, a)\right\|_{L_{\kappa}^{p}} & \leq \frac{a^{\alpha}}{\rho_{\kappa}}\left\|h^{-\alpha} \omega_{p, \kappa}(f, h)\right\|_{L_{\kappa}^{\infty}\left(\mathbb{R}^{+}\right)} \times \int_{-\infty}^{\infty}\left|z^{\alpha} \psi(z)\right| d \sigma_{\kappa}(z) \\
& \leq \frac{a^{\alpha}}{\rho_{\kappa}}\left\|h^{-\alpha} \omega_{p, \kappa}(f, h)\right\|_{L_{\kappa}^{\infty}\left(\mathbb{R}^{+}\right)} \times\left\|z^{\alpha} \psi\right\|_{L_{\kappa}^{1}(\mathbb{R})} .
\end{aligned}
$$

Next theorem is the converse of the above theorem. The $\kappa$-Hankel wavelet coefficients is sufficient to characterize Besov $\boldsymbol{\kappa}$-Hankel spaces.

Theorem 4.2. Suppose $0<\alpha<1$, and a function $\psi$ is a real $C^{1}$-regular wavelet with first derivative rapidly decreasing. If $f \in L_{\kappa}^{p}(\mathbb{R})(1<p<\infty)$, and if $a^{-\alpha}\left\|\left(\mathcal{H}_{\psi}^{\kappa} f\right)(a, \cdot)\right\|_{L_{\kappa}^{p}} \in L_{\kappa}^{q}\left(\mathbb{R}^{+}, \frac{d a}{a}\right),(1 \leq q \leq \infty)$, then $f \in \mathcal{B H}_{\alpha, \kappa}^{p, q}$ and we have

$$
\begin{gathered}
\left\|h^{-\alpha} w_{p, \kappa}(f, h)\right\|_{L_{\kappa}^{q}\left(\frac{d h}{h}\right)} \leq \frac{M_{\kappa}}{C_{\kappa, \psi} \rho_{\kappa}}\left(\frac{M_{\kappa}+1}{\alpha}\|\psi\|_{L_{\kappa}^{1}}+\frac{M_{\kappa}}{(1-\alpha)}\left\|\psi^{\prime}\right\|_{L_{\kappa}^{1}}\right) \\
\times\left\|a^{-\alpha}\right\|\left(\mathcal{H}_{\psi}^{\kappa} f\right)(a, \cdot)\left\|_{L_{\kappa}^{p}}\right\|_{L_{\kappa}^{q}\left(\frac{d a}{a}\right)}
\end{gathered}
$$

Proof. Let $f \in L_{\kappa}^{p}(\mathbb{R})$. By inversion formula of $\kappa$-Hankel wavelet transform

$$
f(x)=\frac{1}{C_{\kappa, \psi} \rho_{\kappa}} \int_{0}^{\infty} \frac{d \sigma_{\kappa}(a)}{a^{2 \kappa}} \int_{-\infty}^{\infty}\left(\mathcal{H}_{\psi}^{\kappa} f\right)(a, b) \psi_{a, b}^{\kappa}(x) d \sigma_{\kappa}(b)
$$

and

$$
\tau_{h}^{\kappa} f(x)=\frac{1}{C_{\kappa, \psi} \rho_{\kappa}} \int_{0}^{\infty} \frac{d \sigma_{\kappa}(a)}{a^{2 \kappa}} \int_{-\infty}^{\infty}\left(\mathcal{H}_{\psi}^{\kappa} f\right)(a, b) \tau_{h}^{\kappa} \psi_{a, b}^{\kappa}(x) d \sigma_{\kappa}(b) .
$$


122

Ashish Pathak and Shrish Pander

DE GRUYTER

Then

$$
\begin{aligned}
\tau_{h}^{\kappa} f(x)-f(x) & =\frac{1}{C_{\kappa, \psi} \rho_{\kappa}} \int_{0}^{\infty} \frac{d \sigma_{\kappa}(a)}{a^{2 \kappa}} \int_{-\infty}^{\infty}\left(\mathcal{H}_{\psi}^{\kappa} f\right)(a, b)\left\{\tau_{h}^{\kappa} \psi_{a, b}^{\kappa}(x)-\psi_{a, b}^{\kappa}(x)\right\} d \sigma_{\kappa}(b) \\
& =\frac{1}{C_{\kappa, \psi} \rho_{\kappa}} \int_{0}^{\infty} \frac{d \sigma_{\kappa}(a)}{a^{2 \kappa}} \int_{-\infty}^{\infty}\left(\mathcal{H}_{\psi}^{\kappa} f\right)(a, b) a^{-2 \kappa}\left\{\tau_{\frac{h}{a}}^{\kappa} \tau_{\frac{b}{a}}^{\kappa} \psi\left(\frac{x}{a}\right)-\tau_{\frac{b}{a}}^{\kappa} \psi\left(\frac{x}{a}\right)\right\} d \sigma_{\kappa}(b) \\
& =\frac{1}{C_{\kappa, \psi} \rho_{\kappa}} \int_{0}^{\infty} \frac{d \sigma_{\kappa}(a)}{a^{2 \kappa}} \int_{-\infty}^{\infty}\left(\mathcal{H}_{\psi}^{\kappa} f\right)(a, b) a^{-2 \kappa} \mathcal{K}_{\kappa}\left(\frac{b}{a}, \frac{x}{a}, y\right) d \sigma_{\kappa}(b) \\
& \times \int_{-\infty}^{\infty}\left\{\tau_{\frac{h}{a}}^{\kappa} \psi(y)-\psi(y)\right\} d \sigma_{\kappa}(y) \\
& =\frac{1}{C_{\kappa, \psi} \rho_{\kappa}} \int_{0}^{\infty} \frac{d \sigma_{\kappa}(a)}{a^{2 \kappa}} \tau_{a y}^{\kappa}\left(\mathcal{H}_{\psi}^{\kappa} f\right)(a, x) \int_{-\infty}^{\infty}\left\{\tau_{\frac{h}{a}}^{\kappa} \psi(y)-\psi(y)\right\} d \sigma_{\kappa}(y)
\end{aligned}
$$

Taking $L_{\kappa}^{p}$-norm on both side and applying Minkowski's inequality, we have

$$
\begin{aligned}
w_{p, \kappa}(f, h) & =\frac{1}{C_{\kappa, \psi} \rho_{\kappa}}\left\{\int_{-\infty}^{\infty}\left|\int_{0}^{\infty} \frac{d \sigma_{\kappa}(a)}{a^{2 \kappa}} \tau_{a y}^{\kappa}\left(\mathcal{H}_{\psi}^{\kappa} f\right)(a, x) \int_{-\infty}^{\infty}\left\{\tau_{\frac{h}{a}}^{\kappa} \psi(y)-\psi(y)\right\} d \sigma_{\kappa}(y)\right|^{p} d \sigma_{\kappa}(x)\right\}^{\frac{1}{p}} \\
& \leq \frac{1}{C_{\kappa, \psi} \rho_{\kappa}} \int_{0}^{\infty}\left|\frac{d \sigma_{\kappa}(a)}{a^{2 \kappa}}\right| \int_{-\infty}^{\infty}\left|\tau_{\frac{h}{a}}^{\kappa} \psi(y)-\psi(y)\right| d \sigma_{\kappa}(y)\left\{\int_{-\infty}^{\infty}\left|\tau_{a y}^{\kappa}\left(\mathcal{H}_{\psi}^{\kappa} f\right)(a, x)\right|^{p} d \sigma_{\kappa}(x)\right\}^{\frac{1}{p}} \\
& \leq \frac{M_{\kappa}}{C_{\kappa, \psi} \rho_{\kappa}} \int_{0}^{\infty} \frac{d \sigma_{\kappa}(t)}{t^{2 \kappa}}\left\|\left(\mathcal{H}_{\psi}^{\kappa} f\right)\left(\frac{h}{t}, \cdot\right)\right\|_{L_{\kappa}^{p}} \int_{-\infty}^{\infty}\left|\tau_{t}^{\kappa} \psi(y)-\psi(y)\right| d \sigma_{\kappa}(y) .
\end{aligned}
$$

Now

$$
\begin{gathered}
\left\{\int_{0}^{\infty} \frac{d h}{h} h^{-\alpha q} w_{p, \kappa}(f, h)^{q}\right\}^{\frac{1}{q}} \leq \frac{M_{\kappa}}{C_{\kappa, \psi} \rho_{\kappa}} \int_{0}^{\infty} \frac{d \sigma_{\kappa}(t)}{t^{2 \kappa}} \int_{-\infty}^{\infty}\left|\tau_{t}^{\kappa} \psi(y)-\psi(y)\right| d \sigma_{\kappa}(y) \\
\quad \times\left\{\int_{0}^{\infty} \frac{d h}{h} h^{-\alpha q}\left\|\left(\mathcal{H}_{\psi}^{\kappa} f\right)\left(\frac{h}{t}, \cdot\right)\right\|_{L_{\kappa}^{p}}^{q}\right\}^{\frac{1}{q}} \\
=\frac{M_{\kappa}}{C_{\kappa, \psi} \rho_{\kappa}} \int_{0}^{\infty} \frac{d \sigma_{\kappa}(t)}{t^{2 \kappa} \int_{-\infty}^{\infty}\left|\tau_{t}^{\kappa} \psi(y)-\psi(y)\right| d \sigma_{\kappa}(y)} \\
\times\left\{\int_{0}^{\infty} \frac{d a}{a} a^{-\alpha q}\left\|\left(\mathcal{H}_{\psi}^{\kappa} f\right)(a, \cdot)\right\|_{L_{\kappa}^{p}}^{q}\right\}^{\frac{1}{q}} \\
=\frac{M_{\kappa}}{C_{\kappa, \psi} \rho_{\kappa}} \int_{0}^{\infty} \frac{d t}{t^{1+\alpha}} \int_{-\infty}^{\infty}\left|\tau_{t}^{\kappa} \psi(y)-\psi(y)\right| d \sigma_{\kappa}(y) \\
\times\left\{\int_{0}^{\infty} \frac{d a}{a} a^{-\alpha q}\left\|\left(\mathcal{H}_{\psi}^{\kappa} f\right)(a, \cdot)\right\|_{L_{\kappa}^{p}}^{q}\right\}^{\frac{1}{q}}
\end{gathered}
$$




$$
\begin{aligned}
= & \frac{M_{\kappa}}{C_{\kappa, \psi} \rho_{\kappa}} \int_{1}^{\infty} \frac{d t}{t^{1+\alpha}} \int_{-\infty}^{\infty}\left|\tau_{t}^{\kappa} \psi(y)-\psi(y)\right| d \sigma_{\kappa}(y) \\
& \times\left\{\int_{0}^{\infty} \frac{d a}{a} a^{-\alpha q}\left\|\left(\mathcal{H}_{\psi}^{\kappa} f\right)(a, \cdot)\right\|_{L_{\kappa}^{p}}^{q}\right\}^{\frac{1}{q}}+\frac{M_{\kappa}}{C_{\kappa, \psi} \rho_{\kappa}} \int_{0}^{1} \frac{d t}{t^{1+\alpha}} \\
& \times \int_{-\infty}^{\infty}\left|\tau_{t}^{\kappa} \psi(y)-\psi(y)\right| d \sigma_{\kappa}(y)\left\{\int_{0}^{\infty} \frac{d a}{a} a^{-\alpha q}\left\|\left(\mathcal{H}_{\psi}^{\kappa} f\right)(a, \cdot)\right\|_{L_{\kappa}^{p}}^{q}\right\}^{\frac{1}{q}} .
\end{aligned}
$$

Using Lemma 2.1, we obtain

$$
\begin{gathered}
\left\{\int_{0}^{\infty} \frac{d h}{h} h^{-\alpha q} w_{p, \kappa}(f, h)^{q}\right\}^{\frac{1}{q}} \leq \frac{M_{\kappa}}{C_{\kappa, \psi} \rho_{\kappa}}\left(\left(M_{\kappa}+1\right)\|\psi\|_{L_{\kappa}^{1}} \int_{1}^{\infty} \frac{d t}{t^{1+\alpha}}+M_{\kappa}\left\|\psi^{\prime}\right\|_{L_{\kappa}^{1}} \int_{0}^{1} \frac{d t}{t^{\alpha}}\right) \\
\times\left\|a^{-\alpha}\right\|\left(\mathcal{H}_{\psi}^{\kappa} f\right)(a, \cdot)\left\|_{L_{\kappa}^{p}}\right\|_{L_{\kappa}^{q}\left(\frac{d a}{a}\right)} \\
=\frac{M_{\kappa}}{C_{\kappa, \psi} \rho_{\kappa}}\left(\frac{M_{\kappa}+1}{\alpha}\|\psi\|_{L_{\kappa}^{1}}+\frac{M_{\kappa}}{(1-\alpha)}\left\|\psi^{\prime}\right\|_{L_{\kappa}^{1}}\right) \\
\times\left\|a^{-\alpha}\right\|\left(\mathcal{H}_{\psi}^{\kappa} f\right)(a, \cdot)\left\|_{L_{\kappa}^{p}}\right\|_{L_{\kappa}^{q}\left(\frac{d a}{a}\right)}
\end{gathered}
$$

Corollary 4.3. Let $f \in \mathcal{B H}_{\alpha, \kappa}^{p, q}(\mathbb{R})(p, q>1,0<\alpha<1)$, then

$$
\|f\|_{\mathcal{B H}_{\alpha, \kappa}^{p, q}}^{p}=\left.|| f\right|_{L_{\kappa}^{p}(\mathbb{R})}+|f|_{\mathcal{B H}_{\alpha, \kappa}^{p, q}}
$$

where $|f|_{\mathcal{B} \mathcal{H}_{\alpha, \kappa}^{p, q}}$ is equal to

$$
|f|_{\mathcal{B} \mathcal{H}_{\alpha, \kappa}^{p, q}}^{q}=\int_{0}^{\infty}\left(h^{-\alpha} w_{p, \kappa}(\phi)(h)\right)^{q} \frac{d h}{h} \approx \int_{0}^{\infty}\left[a^{-\alpha}\left\|\mathcal{H}_{\psi}^{\kappa}(., a)\right\|_{L_{\kappa}^{p}}\right]^{q} \frac{d a}{a} .
$$

Acknowledgement: The research of the second author is supported by University Grants Commission ( UGC), grant number: F.No. 16-9 (June 2019)/2019(NET/CSIR), New Delhi, India.

\section{References}

[1] S. Ben Saïd, A product formula and a convolution structure for a k-Hankel transform on R. J. Math. Anal. Appl. 463 ,no. 2,(2018),1132-1146.

[2] S. Ben Saïd, M.A. Boubatra, M. Sifi, On the deformed Besov-Hankel spaces. Opuscula Math. 40, no. 2,(2020),171-207.

[3] O.V. Besov, On some families of functional spaces. Imbedding and extension theorems. (Russian) Dokl. Akad. Nauk SSSR 126,(1959),1163-1165.

[4] O.V. Besov, Investigation of a class of function spaces in connection with imbedding and extension theorems. (Russian) Trudy. Mat. Inst. Steklov. 60,(1961),42-81.

[5] J.J. Betancor, L. Rodríguez-Mesa, On the Besov-Hankel spaces. J. Math. Soc. Japan 50, no. 3,(1998),781-788.

[6] M. Frazier,B. Jawerth, Decomposition of Besov spaces, Indiana Univ. Math. J. 34, no. 4,(1985),777-799.

[7] D.V. Giang, F. Móricz, A new characterization of Besov spaces on the real line. J. Math. Anal. Appl. 189, no. 2,(1995),533-551.

[8] H. Mejjaoli, K. Trimèche, k-Hankel two-wavelet theory and localization operators. Integral Transforms Spec. Funct. 31, no. 8,(2020),620-644.

[9] Ashish Pathak, Dileep Kumar, Besov-Hankel norms in terms of the Continuous Bessel wavelet transform, arXiv:2012.01354.

[10] V. Perrier,C. Basdevant, Besov norms in terms of the continuous wavelet transform. Application to structure functions. Math. Models Methods Appl. Sci. 6, no. 5,(1996), 649-664.

[11] E.M. Stein, Singular integrals and differentiability properties of functions, Princeton University Press,(1970).

[12] E.M. Stein, Harmonic analysis: real-variable methods, orthogonality, and oscillatory integrals, Princeton University Press, (1993). 
[13] A. Zygmund,Trigonometric series: Vols. I, II. Second edition, reprinted with corrections and some additions Cambridge University Press, London-New York, (1968). 